\title{
Ueber verschiedene, auf deutsche Staphylinen bezügliche Bemerkungen des Herrn Fauvel
}

von

Dr. G. Kraatz.

1

ch habe mehreren Bemerkungen gegenüber, welche im Laufe der letzten Jahre von H. Fauvel und Anderen über einige von mir aufgestellte Gatlungen und Arten gemacht worden sind, geschwiegen. einerseits weil ich hoffen durfte, dieser oder jener Irrthum würde von anderer Seite berichtigt werden, andrerseits weil wiederholte kleinere Berichtigungen leicht den Eindruck einer gewissen Rechthaberei hervorrufen können. Eine solche liegt mir, glaube ich, fern; man würde aber berechtigt sein, dieselbe vorauszusetzen, wenn ich in dem Verzeichnisse der deutschen Käfer eine Anzahl von Bemerkungen, welche Herr Fauvel gemacht hat, unbeachtet liefse, ohne meine Gründe dafür anzugeben.

\section{Ueber die Gattung Stenusa Kraatz.}

Herr Fauvel erklärt sich (Annal. de France 1861 p. 89) gegen die Aufrechterhaltung meiner Gattung Stenusa, gegen welche auch schon Du Val sich ausgesprochen habe. Du Val verstand zur Zeit als er die Aleocharinen für sein Genera bearbeitete, von der Systematik dieser schwierigen Gruppe äufserst wenig und hat sich in der Darstellung der Mundtheile die gröbsten Fehler zu Schulden kommen lassen, wie ich in der Besprechung seines Werkes (Berl. Entomol. Zeitschrift III. p. v.) ausführlich nachgewiesen habe. Du Val's Urtheil kann also nicht für mich maafsgebend sein.

Wenn H. Fauvel die Silusa und Stenusa als ,deux insectes de fasciès et de moeurs analogues": bezeichnet, so kann ich ihm nicht beweisen, dafs sie keine habituelle Aehnlichkeit besitzen, wenn für ihn eine solche existirt; ob dieselben aber für unsere deutsche 
Entomologen, die ihre Augen für Slaphylinen geübt haben, vorhanden ist, erscheint mir zweifelhaft; für mich war und ist bei diesen Käfern grofse habituelle Verschiedenheit vorhanden. Von analoger Lebensweise beider Gattungen kann doch nicht gut gesprochen werden, da ich die Stenusa rubra stets in Pilzen angetroffen babe, die Silusa noch nie. Ein Hauptgrund für die Aufstellung zweier Gattungen ist hier die verschiedene Bildung des Kinns gewesen, ein Umstand welcher denjenigen. die sich um streng wissenschaftliche Principien bei der systematischen Eintheilung nicht kümmern oder den Werth der verschiedenen Bildungen für die Systemalik nicht studirt haben, lächerlich erscheinen mag. Wenn bei hunderten von Homaloten die Kinnbildung dieselbe ist, aber schon bei den zwei angeblich habituell ähnlichen früheren Silusa-Arten eine recht abweichende, so ist eben hinreichender Grund zur Aufstellung zweier Gattungen vorhanden. Was soll die Phrase: Où M. Kraatz se serait-il arrêté, s'il avait pris pour base le mème criterium dans l'étude de Homalota? Gerade der Umstand, dafs ich die natürliche Gattung Homalota nicht zersplittert habe, spricht dafür, daf's ich mit Kritik gearbeitet habe.

Dafs H. Fauvel den Werth, welchen die Bildung der Unterlippe für die natürliche Systematik besitzt, nicht kennt, habe ich auch schon früher (Berl. Ent. Zeitschr. 1866 p. 416., 4.) nachgewiesen, als es sich darum handelte zu erklären, dal's meine Gattung Ischnoglossa aufrecht zu erhalten und nicht mit Stichoglossa Fairm. (Stenoglossa K raatz) zu vereinigen sei; so wie, dafs Westwood's Gattung Encephalus nicht mit Gyrophaena vereinigt werden könne.

\section{Ueber die Gattungen Lomechusa und Atemeles.}

In seiner Enumération des insectes recueillies en Savoie (Bullet. de la soc. lin. de Normandie 1865.) spricht sich H. Fauvel (p. 36. Note 1) für die Vereinigung der Gattungen Lomechusa und Atemeles aus, im Anschlufs an Jaquelin da Val (Gen. II. 10) und Brisout (in Grenier's Cat. d. Col. d. France). Es ist dies eine durchaus unzulässige Concession an den sogenannten Habitus; wer elwas von der Bedeutung der Mundıheile für die Systematik versteht und die verschiedene Bildung derselben bei beiden Gattungen vergleicht, mufs entweder hier zwei Gatlungen annehmen (was auch bei näherer Prüfung der morphologischen Merkmale nothwendig ist) oder consequent darauf verzichten, überhaupt die Mundtheile für die Systematik zu verwerthen, d. h. das einzige sichere Mittel aufgeben, 
welches eine nalurgemäfse Systematik ermöglicht, wenn man es überhaupt anzuwenden gelernt hat.

Bei dieser Gelegenheit will ich bemerken, dafs die von mir in der Naturgesch. d. Ins. Deutschlands Bd. II. p. 116 als Atemeles paradoxus var. major? aufgeführte, in der Berl. ent. Zeitschr. V. p. 412 auf Lomechusa pubicollis Brisout bezogene dritte deutsche Atemeles-Art $\mathrm{n}$ ich $\mathrm{t}$ mit der Lomechusa inflata $\mathrm{Z}$ etterst. identisch, letztere vielmehr eine, wie es scheint, sehr seltene nordische Art ist, welche Thomson wohl mit Recht zu Lomechusa zieht. Für diese Vereinigung sprechen in meinen Augen namentlich die antennae articulis 4-10 subnodosis. Da der prothorax lateribus parce breviter pilosellis und das abdomen segm. 7-o apice emcrginato, welche Thomson in der Gattungsdiagnose von Lomechusa aufführt, bei meinem Atem. pubicollis fehlen, und da der Hinterleib desselben nicht gut sublaevis genannt werden kann etc., scheint es mir ganz sicher, dafs Lomech. inflata Zett. Thoms. nicht mit Atemeles pubicollis identisch sein kann. Ich halte es übrigens wohl für möglich', dafs diese letztere Art auch in Schweden vorkommt; H. Fiseher hat sie bei Berlin in den letzten Jahren in Mehrzahl aufgefunden; süddeutsche Stücke erhielt ich von den Herren Scriba und Prof. Doebner; Stücke aus Dr. Schneider's Sammlung stammen wahrscheinlich aus Oesterreich; auch besitze ich Stücke aus Serbien von Zebe.

\section{Ueber die Gattung Crataraea Thomson.}

Herr Fauvel wünscht (Bull. Soc. Lin. Norm. 1865.), daf's ,en vertu du droit de priorité‘ im Grenier'schen Cataloge dem Gattungsnamen Crataraea Thoms. die Priorität eingeräumt werde vor dem Gattungsnamen Haploglossa Kraatz. Wie Herr Fauvel dies Prioritätsrecht begründen will, isł mir nicht recht klar, da der Autor der Gattung Crataraea meine Gattung Haploglossa vor seiner Gattung Crataraea selbst mit zwei Arten aufführt (Scand. Col. II. p. 281), und bei der zweiten, der Haploglossa rufipennis, mich citirt („Kraatz, Naturg. d. Ins. Deutschl. II. 81. 3.“). Die Gattung Crataraea wird von Thomson auf Haploglossa suturalis errichtet, welche ich zu Haploglossa gestellt habe. Da es mir augenblicklich an Zeit fehlt zu prüfen, ob die Gattung Crataraea haltbar ist, die Art aber in der Färbung recht abweichend von den anderen $\boldsymbol{H}_{\boldsymbol{y}}$ ploglossa-Arten erscheint, so mag Crataraea einstweilen als Untergatlung von Haploglossa aufgefafst werden.

Der Name Haploglossa ist übrigens von mir bereits früher 
(Berl. Ent. VI. p. 300) in Microglossa umgeändert worden, weil der Gattungsname schon vergeben war.

\section{Ueber die Gattung Cyphea Fauvel.}

In der Naturgeschichte der Insekten Deutschlands II. p. 191 habe ich von der Oxypoda curlula Er. (als mir nur das cinzige typiscbe Exemplar auf dem Berliner Museum bekannt war, dessen Mundtheile ich nicht unlersuchen konnte) gesagt: höchst wahrscheinlich gehört der Käfer vicht zu dieser Gattung, sondern zu den Euryusen.

Herr Fauvel hat nun Exemplare des Käfers von verschiedenen Punkten Frankreichs untersucht und sich veranlafst gefunden, auf dieselben die Gattung Cyphea zu gründen (Annal. de la soc. ent. de France 1863 pag. 219). Obwohl die Beschreibung derselben mit den für mich schmeichelhaften Worten eingeleitet wird: ,notre savant collègue jugeait alors avec la perspicacité que chacun lui connait, et aujourd'hui que, plus heureux que Mr. Kraatz, j'ai pu recourir aux rélévations du microscope, c'est avec un bien vif plaisir que je déclare que de tous les Aleochariens connus, ce sont en effet les Euryusa, qui se rapprochent le plus génériquement de l'espèce d'Erichson," so mufs ich zu meinem Bedauern gestehen, dafs ich jetzt meine frühere Vermuthung nur noch zum Theil für richtig erkläre, die Aufstellung der Gattung Cyphea aber nicht für gerechtfertigt halten kann.

Als ich vor längerer Zeit ebenfalls in den Stand gesetzt war, die Mundtheile einer Oxypoda curlula Er. microscopisch zu untersuchen, erkannte ich sofort an der eigenthümlichen Gestalt der Zunge ') und der Lippentaster, dafs der Käfer zur Gattung Placusa gehöre. Nachdem ich jetzt die Beschreibung der Gattung Cyphea nochmals sorgfältig geprüft habe, glaube ich mich früher nicht getäuscht, sondern richtig gesehen zu haben. Zwar sagt H. Fauvel: ,Les Cyphea sont plus voisines des Euryusa que d'aucun autre genre, mais il serait toujours facile des séparer etc.", in-

1) Es erscheint mir auffallend, dafs $\mathrm{H}$. Fauvel, wenn er die verschiedenen Bildungen der Unterlippe genau studirt hat, uns z. B. bei seiner Gattung Arena die Abbildung derselben schuldig bleibt, nachảem er hundert Stück davon gefangen und im Stande gewesen war ${ }_{2}$ en sacrifier une série aux communes exigences du microscope et du crayon." Die Abbildung der Zunge ist aber um so wichtiger als sie sich schwer beschreiben läfst. 
dessen läl'st sich aus seiner Beschreibung nur nachweisen, dafs keine Verwandtschaft mit Euryusa vorhanden ist, und dafs jedenfalls die Verwandtschaft mit Placusa (an die H. Fauvel gar nicht gedacht hat) eine viel grölsere ist, als mit Euryusa.

Meine Gattungsdiagnosen von beiden Gattungen (Naturgesch.d. Ins. Deutschl. II. p. 71 und p. 329) lauten:

Maxillae mala interiore intus spimulis sensim longioribus ciliata

(Euryusa).

\section{- brevioribus ciliata}

(Placusa).

Ligula elongata, angusta, integra (Euryusa).

- brevis, latissima, integra, rotundata (Placusa).

Palpi labiales triarticulati, art. 2 primis arcte connexis (Eur.).

bi articulati, art. $1^{\circ}$ crasso, secundo tenui (Plac.).

Tarsi ant. 4-, posteriores 5 articulati, articulo primo sequentibus paulo longiore (Eur.), arliculo primo elongato (Placusa).

Bei Cyphea ist das erste Glied an den Hinterfüfsen „de la longueur au moins des trois suivants réunis", also ist es viel verwandter mit dem I angen ersten Gliede der Placusa, als dem ersten Gliede bei Euryusa, welches nur et was länger als die folgenden ist.

Die Beschreibung der Lippentaster (paraissant de deux articles à peu près egaux) ist so ungenau, dafs Herrn Fauvel's Erklärung abzuwarten ist, ob sie mit Euryusa oder Placusa übereinstimmen, $d$. h. ob die beiden ersten Glieder an den dreigliedrigen schlanken Lippentastern gleichbreite, mit einander fast verwachsen sind, oder ob das erste Glied an den ziemlich kurzen zweigliedrigen Lippentastern ziemlich stark angeschwollen ist? Letzteres ist um so wahrscheinlicher, als Fauvel sagt, es sei durch die Gestalt der palpes maxillaires robustes, courts leicht von Euryusa zu unterscheiden; kurze gedrungene Maxillartaster bedingen aber in der Regel ähnlich gedrungene Lip pentaster.

Die $Z$ unge wird nun zwar, von $H$. Fauvel allongée, étroite genannt, indessen mag irgend ein sachkundiger Pariser Entomologe entscheiden, ob sie in Wirklichkeit mehr mit meiner Abbildung der kurzen, breiten Zunge von Placusa (Linnaea ent. XI. Tab. I. Fig. 31) übereinstimmt, als mit der schlanken, fadenförmigen Zunge von Euryusa.

Die Placusa-Arten leben, wie ich (Insekten Deutschlands II. pag. 229) angegeben, unter Baumrinden, Cyphea findet sich, ,sous les écorces, principalement celles du Charme", Euryusa lebt bei 
Ameisen. - Cyphea hat "le fasciès analogue à celui des Oxypodes"; die Placusen sind von der Gestalt einer flach gedrückten Homalota oder Oxypoda (Kraalz, Ins, Deutschl. II. p. 329). Hampe hat die Oxypoda curtula sogar als Placusa carbonaria beschrieben, wie ich seiner Zeit nachgewiesen habe.

Nach alledem bleibt mir völlig unersichtlich, wodurch Cyphea den Euryusa näher verwandt sein soll als jeder Aleocharinen-Gattung? Wenn ich aus dem Habitus vermuthete, dafs Oxypoda curtula keine Oxypoda sei, so hatte ich recht; die spätere Untersuchung der Mundtheile zeigte mir sofort, dafs der Käfer, trotz einer gewissen äufseren habituellen Aehnlichkeit mil den Euryusen, eine Placusa sei, womit die Angaben Fauvel's, die ich für richtig halte, wesentlich im Einklang stehen. Sollte H. Fauvel die Richtigkeit meiner Ansicht anerkennen, so würde mir dies um so lieber seiı, als seine Urtheile über mehrere der von mir angenommenen oder aufgestellten Galtungen mir mehr auf Oberflächlichkeit von seiner Seite, als auf Mangel an Kritik meinerseits zu deulen scheinen; er würde dann auch vielleicht erkennen. dafs er auf diesem Gebiete nicht so sicher zu Hause ist, als seine kurzen Urtheile glauben machen könnten.

\section{Ueber Aleochara procera Er. und spadicea.}

H. Fauvel will nach einer längeren Auseinandersetzung (Annal. de France 1863 p. 215 -218) Aleochara procera Er. wieder zu Ocalea gezogen wissen, zu der sie Erichson gestellt hatte. Ich habe die Maxillartaster der Ocalea procera Erich. seiner Zeit genau untersucht, weil mir die Art nach dem Habitus nicht zu dieser Gattung zu gehören schien, sondern zu Aleochara, und habe sie fünfgliedrig wie bei dieser Gattung gefunden, während alle übrigen Aleocharinen-Gattungen nur 4 gliedrige Maxillartaster besitzen. Dieser Umstand scheint mir mehr dafür zu sprechen, dals die procera zu Aleochara gehört als der, dafs Fauvel das fünfte Glied nicht gesehen hat. Derselbe beansprucht gewissermalsen noch einen durch das Microscop unverdorbenen, richtigeu Blick für die Stellung der Thiere nach dem Habitus zu haben, denn er sagt: „enfin, le fasciès trop souvent négligé peut-ètre des adeptes du mieroscope - n'est pas différent de celui des Ocalea el l'éloigne au contraire beaucoup de celui des Aléochares:. Wie soll ich nun aber anders zur besonderen Untersuchung der Mundtheile der OcaLea procera Er. gekommen sein, als weil mir ihr fasciès verschie- 
den von dem der Ocalea erschien? Wäre dies nicht der Fall gewesen, so hätte ich die Ocalea procera gar nicht untersucht.

Herr Fauvel scbliefst: ,je suis donc porté à conclure à la restitution des deux espèces d'Erichson parmi les Ocalea, restitution non moins justifiée par leurs moeurs que par leurs caractères génériques". Welches sind denn nun die Sitten der Ocalea procera und spadicea? Von Herrn Fauvel hören wir nur: ,je recueillis, il y a quelques années, dans une forêt de Calvados, un certain nombre d'exemplaires d'une Ocalea identique avec deux 0 . procera d'Allemagne. Von der spadicea heifst es: ,je laisse de côté spadicea qui est très voisine de procera et dont je ne connais pas les types". Das ist die ganze Sittenschilderung, durch welche Herr Fauvel sich für berechtigt hält, die beiden Arten zur Gattung Aleochara und nicht zu Ocalea zu stellen. I ch kann als die einzige bekannte Sitte für beide nur die angeben, an vereinzelten Localitäten äufserst selten zu sein, mufs es dabei zugleich als gefährlich bezeichnen, dergleichen Phrasen einzuführen und sie zur Unterstützung von wissenschaftlichen Argumentationen zu benutzen; ich würde sie auch, wie bisher, ignorirt haben, wenn der Erfoly nicht der wäre, dafs von ihnen in den Catalogen Notiz genommen wird, wenn sie unwiderlegt bleiben.

\section{Ueber den Gattungsnamen Heterops.}

Herr Fauvel corrigirt (Bull. Soc. Lin. Norm. 1865 extr. p. 15) den Grenier'schen Catalog dahin, dafs er sagl: „Le nom proposé par Eschscholz (Heterhops) doit prévaloir (vor Amphichroum K ra a tz) comme plus ancien, und verweist dabei auf Bull. de Moscou 1860 II. 648. Dadurch macht sich H. Fauvel zum Nachbeter von Motschulsky, ohne Motschulsky's Angabenzu prüfeu, die, wie gewöhnlich, auch hier nur unnütze Verdächtigungen und nicht das Richtige geben.

Motschulsky sagt a. a. O.: „Une seconde espèce de ce genre se trouve dans les possessions russes en Amérique et a été citée par le comte Mannerheim comme genre particulier sous le nom de Heterops Esch b. dans ce Bulletin 1852 p. 98 et décrit en 1843 No. 2 p. 234 comme Arpedium. M. Kraatz qui parle de cette dernière deseription, se garde bien de citer le nom générique appliqué à cette espèce, il y a un quart de siècle par feu Eschscholtz, préférant de lui donner, sans aucune nécessité, un nom de sa propre compositiou. Ce mode de rebaptiser arbitrairement les anciens noms 
ne peut-ètre souflert et chaque entomologiste consciencieux se gardera bien d'introduire de pareilles prétentions dans la science":

Hierzu ist zuerst zu bemerken, dafs die citirte pag. 98 des Bulletin 1852 zu einem Aufsatz von $\mathrm{Chaudoir}$ gehört, dafs dagegen ein Heterops testaceus von Mannerhein auf pa. 380 aufgezählt und durch den Zusatı A. 125 auf Mannerheim's Beschreibung No. 125 im Beitrag zur Käferfauna der Aleutischen Inseln (Bullet. 1843 No. II. p. 234) verwiesen wird. Daselbst ist von Mannerheim ein Arpedium testaceum Eschsch. beschrieben und darunter citir t: Heterops testaceus Eschseh. in litt. Wenn nun Mannerheim für gut befindet 1843 einen Käfer als Arpedium zu beschreiben und 1852 als Heterops aufzuzählen, so mufs man ein Motschulsky sein, um für einen solchen Namen eine Priorität in der citirten Weise zu beanspruchen und die Verdächtigung daran anzuknüpfen: Mr. Kraatz se garde bien etc. Ich konnte Arpedium teslaceum doch nur unter dem Namen citiren, unter dem es der Autor Mannerheim beschrieben, und brauchte den Eschscholtz'schen Namen in litleris doch nur anzuwenden wenn er mir passend schien, oder ich das Citat überhaupt bemerkt hatte. Dann hätte die Gattung Heterops Kraatz heifsen könuen, aber doch niemals Heterops Eschsch., da man eine Gattung nicht wissenschaftlich dadurch begründet, dals man einem Käfer aus einer bekannten Gattung in seiner Sammlung einen anderen Gattungsnamen anhängt. Mein Gattungsname Amphichroum braucht also wohl nicht dem von Heterops oder hops, wie $\mathrm{H}$. Fauvel schreibt, Platz zu machen. Dagegen darf ich wohl erwarten, dafs ,chaque entomologiste consciencieux se gardera bien d'introduire, dans la science de pareilles prétentions" eines Motschultsky, und das giftige Geschwätz desselben, ohne ihren Werth genau geprüft zu haben.

\section{Ueber Bledius tricornis Herbst und Verwandte.}

In seiner Enumération des insectes recueillis en Savoie p. 59 (Bullet. de la société Linéenne de Normandie) giebt H. Fauvel eine Uebersicht der mit Bledius tricornis Herbst verwandten Arten and erklärt meinen Bledius spectabilis (Naturgesch. d. Ins. Deutschl. II. p. 821) für eine südliche Varietät des tricornis Herbst; zugleich giebt er an, dafs sich aus meiner Beschreibung des letzteren leicht ergäbe, dafs ich beide Arten confundirt habe. Nun heifst es bei meinem spectabilis: weit gröfser und durchgängig weitläufiger punktirt als tricornis, der Seitenrand der Stirn beim $\delta$ nur zu einem stumpfen Höcker erhoben; vom tricornis heifst es: beim $\sigma^{\circ}$ ist die Stirn je- 
derseits zu einem langen Horn erhoben. Diese Bezeichnungen schliefsen eine Confundirung beider Arten in der Beschreibung vollständig aus und ist mir eine solche auch sonst nicht ersichtlich. In den Käfern der Mark (I. p. 578) sagt Erichson vom tricornis б': die Seitenränder der Stirn über der Einlenkung der Fühler zu einem zusammengedrückten zugespitzten Horn erhoben, mithin hat er deutlich den märkischen tricornis beschrieben. In seinen Genera et spec. Saphylinorum dagegen (p. 764) heifst es vom tricornis $\delta$ : fronte lateribus supra antennarum basin acute angulato-elevato. Danach scheint es, dafs er hier meinen spectabilis vor sich gehabt, und mit tricornis Herbst verwechselt hat. ') In Folge dessen unterscheiden Mulsant et Rey (Opuscules entom. XII. 1861 p. 165) vom Bledius spectabilis Erichson einen Bledius nuchicornis nov. spec., welcher von Versailles stammt und von Mulsant mit Sicherheit auf den tricornis Olivier von Paris bezogen wird; dieser nuchicornis ist jedenfalls nichts anderes als der tricornis Erichs. (Col. March.), Kraatz (Ins. Deutschl.), und Mulsant unterscheidet seinen nuchicornis gerade so wie ich meinen spectabilis und tricornis Herbst unterscheide, denn er sagt am Schlufs: le nuchicornis diffère du tricornis Er. par sa taille plus petite et par les cornes frontales du $\sigma$ beaucoup plus longues.

Da der Bledius spectabilis bis jetzt noch nicht in Deutschland aufgefunden ist, so ist kein Grund für mich vorhanden, ihn auf den Staph. tricornis Herbst zu beziehen; dagegen sind auf diesen der tricornis Er. (Col. March.) und der nuchicornis Muls. so lange zu beziehen, bis $\mathrm{H}$. Fauvel die Identität meines spectabilis etwa mit dem tricornis Herbst nachgewiesen hat. Herr Fauvel spricht nur von einem vrai tricornis, welcher für ihn die südeuropäische, grölsere Art ist.

Den spanischen Bledius Graellsii Fauvel, welcher bei dieser Gelegenheit beschrieben wird, kenne ich nicht; er soll sich durch dichte, runzlige Punktirung von spectabilis unterscheiden.

Hiernach stellen sich die besprochenen Arten übersichtlich so zusammen:

tricornis Herbst, Ol., Krtz.

tricornis Er. (Col. March.) nuchicornis Muls. spectabilis Kraatz.

tricornis Er. (Gen. et Spec.)

tricornis Muls. Fauvel.

1) Wahrscheinlich hat er beide Arten vor sich gehabt, denn er neunt in der Diagnose den thorax crebre punctatus, in der Beschreibung magis minusve crebre punctatus. 
Ueber Stenus glacialis Heer.

H. Fauvel lenkt (Bull. Soc. Lin. Norm. 1865) die Aufmerksamkeit auf den Stenus glacialis Heer, mit welchem nach seiner Ansicht leicht der muscorum Fairm. identisch sein könne etc. Dazu will ich nicht unbemerkt lassen, dafs hinter meiner Diagnose des glacialis (Nat. Ins. Deutschl.) durch einen Druckfehler $1 \frac{1}{2}$ lin. statt $2 \frac{1}{2}$ lin. Länge angegeben ist. Allerdings heifst es darauf: nicht unbedeutend gröfser als Stenus impressus.

Ueber Anthobium Rhododendri Baudi.

Anthobium Rhododendri Baudi wird von Fauvel (Bull. soc. Lin. Norm. 1865) mit Recht als eine von sorbi verschiedene Art (mit zwei Längseindrücken auf dem Thorax und winkelig zugespitzten Flügeldecken des ठ) angesprochen. Meine Angabe (Nat. Ins. Deutschl II. p. 1018), dafs die mir mitgetheilten typischen Ex. dieser Art zum sorbi gehören, ist richtig, aber es mufs bei der Uebersendung derselben ein Irrthum stattgefunden haben, da sie nicht das wirkliche Rhododendri Baudi sind, wie aus der Beschreibung deutlich hervorgeht, welche ich früher zu vergleichen unterlassen to. 


\section{$2 \mathrm{BHL}$ Biodiversity Heritage Library}

Kraatz, G. 1868. "Ueber verschiedene, auf deutsche Staphylinen bezügliche Bemerkungen des Herrn Fauvel." Berliner entomologische Zeitschrift I herausgegeben von dem Entomologischen Vereine in Berlin 12(3), 339-348. https://doi.org/10.1002/mmnd.18680120316.

View This Item Online: https://www.biodiversitylibrary.org/item/34406

DOI: https://doi.org/10.1002/mmnd.18680120316

Permalink: https://www.biodiversitylibrary.org/partpdf/210138

\section{Holding Institution}

Smithsonian Libraries

\section{Sponsored by}

Smithsonian

\section{Copyright \& Reuse}

Copyright Status: Public domain. The BHL considers that this work is no longer under copyright protection.

This document was created from content at the Biodiversity Heritage Library, the world's largest open access digital library for biodiversity literature and archives. Visit BHL at https://www.biodiversitylibrary.org. 